19 Revue d'histoire du XIXe siècle

Société d'histoire de la révolution de 1848 et des

révolutions du XIXe siècle

$28 \mid 2004$

Religion, politique et culture au XIXe siècle

\title{
La loi de séparation de 1905 ou l'impossible rupture
}

Mathilde Guilbaud

\section{OpenEdition}

\section{Journals}

Édition électronique

URL : http://journals.openedition.org/rh19/627

DOI : $10.4000 /$ rh19.627

ISSN : $1777-5329$

Éditeur

La Société de 1848

Édition imprimée

Date de publication : 1 juin 2004

Pagination : 163-173

ISSN : 1265-1354

Référence électronique

Mathilde Guilbaud, "La loi de séparation de 1905 ou l'impossible rupture », Revue d'histoire du XIXe siècle [En ligne], 28 | 2004, mis en ligne le 19 juin 2005, consulté le 21 décembre 2020. URL : http:// journals.openedition.org/rh19/627 ; DOI : https://doi.org/10.4000/rh19.627

Ce document a été généré automatiquement le 21 décembre 2020.

Tous droits réservés 


\title{
La loi de séparation de 1905 ou l'impossible rupture
}

\author{
Mathilde Guilbaud
}

1 De l'article deux du titre premier de la loi de 1905, on retient trop souvent l'idée que ne « reconnaissant» "aucun culte», la République, c'est-à-dire l'État, est absolument étrangère à la chose religieuse. Le mot même de « séparation »-qui figure dans le titre de la loi, mais ne réapparaît dans aucun de ses articles - évoque en effet l'idée d'une rupture, d'un divorce ${ }^{1}$, d'une dissociation totale entre la chose publique et la chose religieuse, comme si les deux domaines ne devaient avoir aucun rapport et pouvaient évoluer de façon absolument indépendante, dans une ignorance mutuelle qui serait tout à la fois l'expression et la garantie de la laïcité. Dans le langage courant, la «loi de séparation des Églises et de l'État » est souvent présentée comme la loi de « séparation de l'Église et de l'État ». C'est que la séparation concerne surtout l'Église catholique, d'abord en raison de son importance numérique, ensuite, parce que l'on ne reprochait pas aux cultes protestants ou israélite d'être liés à l'État.

2 Certains hommes politiques voulaient une séparation radicale, qui amènerait le gouvernement à ignorer les affaires intérieures de l'Église; ainsi, dans L'Aurore du 21 juillet 1904, Georges Clemenceau résume-t-il d'un mot sa politique à l'égard de l'Église : «Divorçons $»^{2}$. Ce vœu rejoignait celui de certains catholiques, comme le journaliste contemporain Julien de Narfon, qui voyaient dans la séparation le moyen de libérer l'Église des chaînes concordataires ${ }^{3}$. Cependant, d'autres personnalités, inspirées par un "gallicanisme concordataire " ${ }^{4}$, héritier de l'Ancien Régime et de la Révolution française, voulaient abroger le concordat tout en continuant d'intervenir administrativement dans la discipline de l'Église. C'est le paradoxe de la politique d'Émile Combes, critiquée par Clemenceau, qui estimait que le président du conseil Combes voulait « recommencer le concordat sans le pape ${ }^{5}$.

3 L'élaboration de la loi peut être lue comme une lutte entre ces deux conceptions de la séparation : à un projet libéral, visant à donner à l'Église son autonomie, s'oppose un projet, anticlérical et gallican, qui maintiendrait l'Église sous la surveillance et le contrôle 
de l'État ${ }^{6}$. Si la vision libérale l'emporte dans le projet final ${ }^{7}$, celui-ci n'en est pas moins le résultat d'un compromis, le fruit d'un des débats les plus longs et passionnés de l'histoire de la Troisième République. Le Concordat était abrogé, mais l'esprit gallican qui l'avait inspiré demeurait malgré tout: dans la nouvelle loi, nombreuses sont les mesures restrictives, dues à la méfiance qu'inspire la liberté de l'Église.

En pratique la séparation ne pouvait être absolue, ne serait ce qu'à cause des questions matérielles à régler. La loi de séparation inaugure en réalité une marche "vers de nouveaux rapports entre l'Église et l'État ${ }^{8}$. Si la rupture est présentée comme "radicale», ce ne peut être que d'un point de vue symbolique et politique. La loi de séparation, loin de rendre l'Église catholique étrangère à l'État, obligera finalement celuici à légiférer plus que jamais, pour établir de nouveaux liens entre les deux institutions. Malgré le décret du 17 avril 1906 qui supprime la direction des cultes, le ministère de l'Instruction publique, des Beaux-Arts et des cultes conserve une administration centrale des cultes jusqu'au 17 août 1911. À cette date, un décret signé par le président du Conseil, Joseph Caillaux, supprime « une administration que le parfait fonctionnement de la loi de séparation des Églises et de l'État rend à présent inutile " ${ }^{9}$; cette mesure demeure toute théorique puisque l'administration continue d'exister, rattachée au ministère de l'Intérieur. Et en 1920, est créé le poste de «conseiller technique pour les affaires religieuses " auprès du ministère des Affaires étrangères ${ }^{10}$, conseiller dont la tâche essentielle est le contrôle du corps épiscopal. Les deux institutions que sont l'Église et l'État continuent donc bien d'entretenir des rapports après la séparation.

11

Une partie de l'opinion catholique ne s'y est pas trompée. En 1905, les Semaines religieuses des diocèses de Meaux et de Versailles présentent le projet relatif à la séparation comme une nouvelle "alliance ", beaucoup trop contraignante pour l'Église. Ces deux bulletins diocésains mettent en garde leurs lecteurs, ces "braves gens", comme les qualifie la Semaine religieuse de Versailles, contre la chimère d'une séparation " neutre » conférant la liberté à l'Église, et la " débarrassant des liens du concordat " ${ }^{12}$. Les " amis de la liberté " ne doivent pas $" s$ 'y laisser prendre $"{ }^{13}$, disent ces organes diocésains, qui dissuadent les catholiques d'entretenir un « fol espoir » : la loi prévue est «abominable » ${ }^{14}$. Ce n'est pas une loi libérale ${ }^{15}$, « c'est une loi de police ! c'est un code pénal ! [...] Seul le défenseur de la morale et le représentant de Dieu doit se taire. Sinon, l'amende, la prison, la fermeture de l'église ! Ah! la belle liberté qu'il faut acheter par la prison $! »{ }^{16}$. On souligne que cette séparation à la française est oppressive, très éloignée de la séparation à l'américaine, qui est valorisée ${ }^{17}$. Votée dans un esprit anticlérical, la loi sera la suite logique et l'aboutissement de la longue série de persécutions lancées contre l'Église depuis 1880 . Désormais, ajoutent les bulletins, l'oppression subie par les catholiques va s'accroître: "C'est une union à rebours qui va se refaire après la séparation, l'union dans la servitude et la persécution. [...] Non, il n'y aura pas plus de paix que de liberté après la séparation » ${ }^{18}$. Sont particulièrement dénoncées les atteintes aux grands principes républicains : la liberté de conscience ${ }^{19}$ et l'égalité devant la loi ${ }^{20}$.

6 Tyrannique, la loi de séparation établit un nouveau contrat inacceptable. Les Semaines religieuses craignent la publication des "articles organiques de la loi de séparation", comme il y avait eu ceux du Concordat, qui avaient retranché « le peu de liberté » accordé par Bonaparte. Ces nouveaux articles organiques aggraveraient «les restrictions et les prohibitions " déjà dénoncées du temps du régime concordataire ${ }^{21}$. Aussi, dans les semaines précédant le vote de la loi, les organes diocésains estiment-ils qu'il faut refuser 
la Séparation, qu'ils voient comme " un tout petit concordat, dix fois pire que l'ancien » ${ }^{22}$, destiné à faire des catholiques, non plus des obligés de l'État, comme en 1802, mais des esclaves : la loi, qui promet la séparation, établira en réalité « la servitude » ${ }^{23}$. À Meaux, en 1909 encore, on utilise cette image d'un nouveau concordat, pour refuser d'avaliser ce "Concordat de la Séparation », qui ne vise qu’à « replacer l'Église sous le joug du pouvoir civil ${ }^{24}$.

Dans les années qui suivent le vote de la loi et jusqu'à l'Entre-deux-guerres, l'Église catholique refuse absolument de participer à l'application de la loi de séparation, dont elle continue à condamner le principe même. En 1909, quand Aristide Briand, devenu chef du gouvernement, témoigne de sa volonté de pacification, la Semaine de Meaux dénonce le "piège du statut légal » ${ }^{25}$. Tout en s'étant adaptée au nouvel état des choses, l'Église préfère rester dans une certaine illégalité, voire se complaire dans la persécution, manifestant ainsi son opposition à une loi qu'elle n'a pas acceptée : «implorer du Bloc un "statut légal" pour utiliser les édifices qu'ont bâtis et que nous ont légués nos pères, ne serait-ce pas lacérer de nos propres mains nos titres de propriété? ${ }^{26}$. La grande insistance de la Semaine de Meaux sur le sujet révèlerait-elle une certaine propension des catholiques du diocèse à accepter la loi ? En 1910, lors de son arrivée dans le diocèse, le nouvel évêque de Meaux, Mgr Marbeau, cite Pie X pour confirmer l'opposition catholique à la loi : «l'Église a refusé, au prix même de la pauvreté, de laisser toucher en elle à l'œuvre de Dieu. On lui a donc pris ses biens, elle ne les a pas abandonnés ${ }^{27}$. Au printemps 1910, alors que des rumeurs rapportent des tractations entre l'Église de France et le gouvernement, la Semaine religieuse du diocèse de Meaux s'oppose à un «concordat Briand ${ }^{28}$ conclu sans consultation préalable du Saint-Siège. Le bulletin nie farouchement toute possibilité d'accord, en rappelant que les deux seuls signataires d'un Concordat ne peuvent être que le Pape ou son représentant officiel d'une part, le gouvernement français d'autre part; or le chef de l'Église n'a même pas été consulté. Campant sur des positions intransigeantes, le bulletin désavoue les "députés libéraux » qui consentiraient à traiter de la pacification sur la base de la séparation (en acceptant la séparation comme un " problème enterré ») et refuse de les considérer comme des alliés 29. Dans le même ordre d'idées, on montre que l'on peut refuser tout simplement la loi de séparation, comme les viticulteurs du Midi ont refusé la loi supprimant le privilège des bouilleurs de cru, ce qui a conduit à l'abrogation de la loi ${ }^{30}$.

8 Mais comment des catholiques ont-ils pu imaginer que les relations entre la République et l'Église empireraient après la loi de séparation? En effet, celle-ci n'est pas venue briser des relations harmonieuses, mais plutôt sanctionner des difficultés croissantes de fonctionnement, notamment depuis l'Affaire Dreyfus. Le gallicanisme traditionnel s'est transformé à partir de 1880 pour devenir, au début du XXe siècle, un "pseudogallicanisme », un « anti-christianisme » du gouvernement qui organise la " persécution légale $"{ }^{31}$ des catholiques. Dans ces conditions, le processus de séparation ne pourrait-il pas permettre de passer d'une mésentente à un apaisement entre les deux grands corps que sont l'État et l'Église?

9 Il en sera finalement ainsi, mais ce n'est pas la loi de 1905 elle-même qui produira ce résultat. En effet, passées les années de lutte, s'instaurera, grâce aux divers aménagements de la loi, une pacification progressive, qui triomphera avec l'union sacrée en 1914. Et c'est dans les années qui suivirent la séparation que se contractualiseront peu à peu de nouveaux rapports entre l'Église et l'État, pour aboutir à ce que le canoniste Dominique Le Tourneau désigne comme « une sorte de concordat », en tout cas un modus 
vivendi qui s'est progressivement établi par une série d'actes officiels, entre 1907 et $1924^{32}$ . La loi de 1905 n'est donc qu'un des éléments, lui-même remanié plusieurs fois, parmi ceux qui règlent les relations entre l'Église catholique et l'État français. Le concordat a laissé la place à l'édification progressive d'un «statut légal du Culte catholique en droit français " ${ }^{33}$, à la construction d'un compromis durable entre l'Église et l'État.

Avant d'en arriver à cet apaisement, bien des troubles et des antagonismes éclateront, notamment à propos des associations cultuelles, mentionnées dans les articles 4, 18, 19 de la loi, dont elles sont un dispositif central. Leur composition est déterminée de manière paradoxale ${ }^{34}$ : tout en stipulant dans son article quatre que les associations doivent «se conformer aux règles d'organisation générales du culte dont elles se proposent d'assurer l'exercice ", la loi omet la prééminence de la hiérarchie dans l'Église catholique. Pour l'Église, le principe d'autorité n'est donc pas suffisamment reconnu.

11 Au début de l'année 1905 pourtant, malgré des réserves ${ }^{35}$, la majorité des évêques, les notables laïcs, les catholiques libéraux penchaient pour l'acceptation des associations cultuelles ${ }^{36}$, comme en témoignent les nombreux articles que les Semaines religieuses consacrent à leur analyse. Deux raisons jouent alors en faveur de l'acceptation des associations cultuelles : tout d'abord, celles-ci représentent le seul moyen pour l'Église de conserver ses biens matériels; ensuite, la majorité des catholiques veut éviter une opposition au gouvernement qui pourrait aller jusqu'à la guerre civile ${ }^{37}$. Les catholiques français apparaissent résignés devant l'inéluctabilité de la séparation, et désireux d'un divorce « à l'amiable ». À Meaux, l'évêque lui-même semble avoir prévu la constitution de ces associations dans le diocèse. Mgr de Briey, évêque de Meaux, fut en effet un des deux seuls dignitaires ${ }^{38}$ à approuver le principe même de la séparation, lors de la première assemblée des évêques de France, au mois de mai 1906, en pleine querelle des inventaires.

Mais l'opinion des catholiques évolue lentement au cours de l'année 1905, et ces changements sont perceptibles dans les bulletins diocésains qui se préoccupent du risque de schisme ${ }^{39}$, de la trop grande place des laïcs ${ }^{40}$, risquant de déboucher sur un «laïcisme» confinant au "protestantisme ${ }^{41}$. En 1906, alors que des catholiques libéraux, comme Julien de Narfon ou les «cardinaux verts " ${ }^{42}$, militent encore pour l'adoption des associations cultuelles, le Saint-Siège prend position. Le souverain pontife choisit la lutte et s'oppose ainsi directement aux catholiques conciliateurs. L'encyclique Vehementer nos du 11 février 1906 conserve une part d'incertitude, mais, le 10 août 1906, la condamnation absolue des associations cultuelles est très claire dans l'encyclique Gravissimo officii. L'Église de France, qui penchait jusque-là dans l'ensemble pour l'acceptation, suit Pie X avec toute la docilité requise. Alors le refus de la loi et de toute accommodation devient absolu, et un certain esprit de martyre tend à s'installer. Mais, de fait, cette réaction intransigeante oblige le gouvernement, qui veut aplanir toutes les difficultés et éviter tout risque de guerre civile, à établir de nouvelles dispositions législatives.

13 En refusant la loi, l'Église a perdu la disposition des édifices du culte et la propriété des biens des établissements ecclésiastiques. Le gouvernement entérine cette décision et laisse l'Église perdre son patrimoine ${ }^{43}$. À l'expiration du délai d'un an prévu pour la constitution des associations cultuelles, les biens des établissements ecclésiastiques supprimés sont mis sous séquestre, pour être dévolus par la suite à des bureaux de bienfaisance. Les églises fabriciennes, construites entre 1802 et 1905, deviennent propriété communale par la loi du 13 avril 1908, qui modifie les titres deux et trois de la loi de séparation. En revanche, le gouvernement ne vet pas entériner la deuxième 
conséquence du refus catholique, qui serait de laisser l'exercice du culte se dérouler en dehors de tout cadre légal. En effet, selon la loi de 1905, le culte ne peut être exercé publiquement sans associations cultuelles : si celles-ci n'existent pas, les églises doivent en principe être fermées. Aristide Briand ${ }^{44}$, ministre de l'Instruction publique et des cultes, décide "d'enfermer malgré eux les catholiques dans la légalité » ${ }^{45}$, par une série de nouvelles mesures. Sous son impulsion, le 31 octobre 1906, le Conseil d'État reconnaît que le culte pourra être exercé légalement même sans associations cultuelles ; l'exercice du culte peut entrer dans le cadre de la loi du 30 juin 1881, qui autorisait les réunions tenues sur initiative individuelle, en les assujettissant toutefois à l'obligation d'une déclaration. Pour faciliter l'application de la loi, le $1^{\text {er }}$ décembre 1906, Briand adresse aux préfets une circulaire qui établit que la déclaration préalable pourra être annuelle. D'autre part, la loi du 2 janvier 1907, tout en maintenant la nécessité de la déclaration préalable pour les réunions cultuelles, conserve l'affectation cultuelle des églises, ce qui permet au prêtre d'avoir un droit de jouissance de l'église, soit qu'il y officie comme simple occupant, soit qu'il ait un droit de jouissance précisé dans un «acte administratif », par exemple un contrat passé avec la commune à laquelle l'église appartient.

14 Selon la déclaration des évêques de France du mois de février 1907, on peut faire l'essai d'une organisation du culte public, à condition d'obtenir un contrat administratif entre maire et curé pour la jouissance de l'église. La Semaine religieuse du diocèse de Meaux publie cette déclaration, qu'elle approuve comme un "véritable traité de paix » proposé par l'Église à l'État. Elle est suivie d'un modèle de procès-verbal de jouissance ${ }^{46}$. Mais les négociations avec le gouvernement achoppent sur la question des responsabilités matérielles de chaque partie pour ce qui a trait aux églises ; l'Église refuse le compromis, et n'obtient donc aucune garantie légale ou exclusivité quant à leur jouissance. Les municipalités, qui en sont les propriétaires, en font ce qu'elles souhaitent. En pratique, les églises restent généralement à la disposition des fidèles. Par exemple, le maire de Melun, qui a refusé de signer les projets de baux proposés par deux curés de la ville, leur accorde des droits d'administration sur leur église - notamment en matière de police et de droit à percevoir des recettes; en contrepartie, les curés doivent payer toutes les dépenses d'entretien et de jouissance, à l'exclusion des grosses réparations et de l'assurance contre l'incendie, et assumer la responsabilité des accidents survenant dans l'église ${ }^{47}$. Au mois de mars 1907, à partir de plusieurs exemples locaux, la Semaine religieuse du diocèse de Meaux montre que « les municipalités sont obligées de laisser les églises à la disposition des catholiques pour le culte public, et de subvenir à leur entretien ${ }^{48}$; elle conclut qu'il ne faut donc pas établir de contrats entre maires et curés, et qu'il est préférable de se contenter de vivre dans le «statu quo " ${ }^{49}$. Dès juin 1905 , la Semaine religieuse du diocèse de Versailles, imaginant les catholiques forcés de louer les églises, recommandait la résistance si nécessaire: "qu'arriverait-il si, [...] en refusant de louer ce qui nous appartient, nous restions dans nos églises jusqu'à ce qu'on nous eut expulsés par la force? [...] Qui sait ? La résistance en bloc ne ferait-elle pas reculer l'ennemi du premier coup ?» ${ }^{50}$. Ici le bulletin diocésain devine que le gouvernement ne pourra empêcher fidèles et clergé d'occuper l'église. À Meaux, on craint quand même, encore en 1907, la confiscation, puisqu'aux termes de la loi, si une association cultuelle ne réclame pas les immeubles avant le 11 décembre 1908, «ils peuvent être désaffectés par décret ${ }^{51}$. Mais l'administration appuie très généralement les catholiques. Ainsi, le 6 janvier 1907, le maire d'Ichy ferme l'église et chasse le curé du presbytère; cette double décision provoque aussitôt la réaction du sous-préfet de Fontainebleau, qui rappelle au magistrat 
municipal que, selon l'article cinq de la loi du 2 janvier 1907, il n'a pas le droit de fermer l'église ${ }^{52}$. Les conseils municipaux, même les plus anticléricaux, sont obligés d'appliquer la loi avec souplesse. D'ailleurs, les 5 et 6 février 1912, la Cour de cassation rend deux arrêts importants, décidant que «les églises catholiques ne peuvent être mises à la disposition que des ministres du culte reconnaissant la hiérarchie catholique et faisant partie de cette hiérarchie ${ }^{53}$. Les églises semblent donc en pratique revenir de droit au culte catholique, sans qu'il y ait de réelle opposition de la part des conseils municipaux. On ne trouve aucune trace de conflit dans le département de Seine-et-Oise, plus conservateur il est vrai ; dans ce département, aucun conseil municipal ne semble donc avoir contesté les droits des catholiques à utiliser les églises.

L'Église continue cependant de résister à toutes les tentatives de conciliation. Les prêtres occupent les églises et se félicitent du droit de jouissance qui leur a été accordé, mais refusent de déclarer les messes, suivant ainsi les instructions de Pie X, qui a interdit d'entrer dans le jeu du gouvernement. Alors, le 11 décembre 1906, en conseil des ministres, Georges Clemenceau ${ }^{54}$, très ferme, invite les parquets à constater les infractions à la loi de 1901, ce qui crée le " délit de messe " ${ }^{55}$. Suivant les instructions pontificales, les Semaines religieuses des deux diocèses étudiés demandent aux curés de célébrer la messe comme auparavant, « en s'abstenant de faire aucune déclaration ». Le bulletin versaillais rassure son clergé sur les contraventions encourues, "purement arbitraires et ne pouvant aboutir qu'à des acquittements ${ }^{56}$. En Seine-et-Marne, où le courant anticlérical est puissant ${ }^{57}$, on sent bien que le climat est différent. Dans un premier temps et à l'insu du clergé, certains catholiques, légalistes, croyant bien faire, ont déclaré auprès des autorités la tenue de réunions publiques dans les églises ${ }^{58}$. Indigné, l'évêque considère que ces laïcs n'ont aucune qualité pour faire des déclarations, d'autant qu'ils contreviennent ainsi aux ordres pontificaux ${ }^{59}$. Ailleurs, des procès-verbaux ont été dressés contre des prêtres ayant célébré la messe sans avoir fait la déclaration préalable ; ce fut le cas à Meaux même, contre le chanoine Gadon, pour la messe du chapitre, et à Saint-Nicolas, contre l'abbé Isambert ${ }^{60}$. Cependant, les condamnations sont légères: ainsi, tel prêtre poursuivi pour non-déclaration et délit de messe fut-il condamné à un franc d'amende ${ }^{61}$. Tout en respectant la lettre de la loi, les tribunaux sont contraints à un esprit de tolérance : comment faire autrement quand aucun prêtre en France ne déclare la messe? Devant l'intransigeance de l'Église, c'est finalement le gouvernement qui cède, constatant l'impossibilité de dresser des procès-verbaux à tous les curés. Dès le 3 février 1907, une circulaire ministérielle ordonne aux préfets de surseoir au relevé des infractions pour délit de messe, puisque, à cette date, un projet de loi abrogeant l'obligation de la déclaration préalable a déjà été adopté par la Chambre des députés. Adopté par le Sénat, ce texte devient la loi du 28 mars 1907 qui consacre le droit de réunion sans déclaration préalable et modifie le titre quatre du décret du 16 mars 1906. $\mathrm{Au}$ mois de mars 1907, on affirme à Meaux, d'après l'avis des jurisconsultes de l'Archevêché de Paris, que les curés ont le droit d'occuper l'église et d'y célébrer le culte ${ }^{62}$ - Une fois de plus, c'est la résistance des catholiques qui a poussé le gouvernement à modifier les termes mêmes de la loi, à s'adapter à la situation.

Des positions radicales de chacune des deux parties, seule l'Église, forte de son unité, a pu tenir la sienne. L'État a été obligé de composer, de négocier pour proposer une solution viable. En réalité, alors que la loi de séparation avait, au moins en partie, l'intention de brimer l'Église, c'est celle-ci qui sort, appauvrie certes, mais victorieuse, du combat entre les deux institutions. Au départ, l'Église semble être vaincue, le gouvernement anticlérical 
est agressif face à des catholiques français tièdes et prêts à tous les compromis. Mais l'intransigeance de Pie X place l'Église de France en position de force vis-à-vis d'un gouvernement qui doit éviter la guerre civile. L'État est obligé de s'incliner devant l'unité catholique : ainsi, une fois votée, la séparation se fait aux conditions que l'Église veut bien accepter. D'après Roger Aubert, c'est l'intransigeance de Pie X qui a contraint le gouvernement à un certain libéralisme, « dont il n'est pas sûr qu'il aurait fait preuve sans la fermeté romaine " ${ }^{63}$. Mais on peut estimer que, dès le départ, certains responsables gouvernementaux, Aristide Briand notamment, étaient ouverts et désireux de trouver un compromis. Ce qui est intéressant, c'est que l'Église catholique ait pu tenir une position intransigeante, alors même que la majorité des catholiques étaient relativement indifférents aux inventaires, plutôt portés à l'acceptation des associations cultuelles et plutôt favorables à la séparation si l'on considère la victoire du Bloc aux élections de mai 1906.

Semaines religieuses reflètent les contradictions idéologiques présentes chez les catholiques. Elles font ainsi preuve, au début de l'année 1905, d'un grand légalisme et d'une volonté d'accepter la loi pour ne pas entrer en conflit avec le gouvernement, tout en exprimant une certaine idéologie contre-révolutionnaire, qui les pousse à considérer la loi de séparation comme une nouvelle constitution civile du clergé. En se référant au cycle historique de la Révolution et du Consulat, elles en viennent à espérer que naîtra un nouveau concordat, présenté comme « la logique de l'histoire " ${ }^{64}:$ « leur loi de séparation ne peut aboutir qu'à une constitution civile du clergé, au schisme, et, par voie de conséquence, à un nouveau concordat ${ }^{65}$. En un mot, les vicaires capitulaires de Versailles présentent la Révolution comme une maladie et le Concordat comme son " remède " ${ }^{66}$. Si la loi de séparation est assimilée à la constitution civile du clergé, peuton considérer que la série d'actes élaborés entre 1906 et 1924 et réglant les rapports entre l'Église et l'État constitue un nouveau concordat? Oui, car si avec la loi de séparation, le gouvernement français a voulu dicter unilatéralement ses conditions à l'Église, Pie X par son intransigeance s'est imposé comme un partenaire obligé. Dans le processus de séparation, l'Église est passée du statut de victime à celui d'acteur. Tenant compte de l'Église, le compromis qui s'est construit après 1905 ne peut-il être considéré comme un concordat puisqu'il s'agit d'une forme d'accord entre les deux partenaires que sont l'Église et l'État?

D'autre part, en ce qui concerne la connotation positive («concorde») du terme de " concordat », Bruno Neveu montre qu'à partir de 1911 un rapprochement s'est opéré entre les deux instances, caractérisé par des «attitudes administratives inspirées par un esprit de plus large compréhension » envers l'Église ${ }^{67}$. Cet état d'esprit est perceptible notamment à travers les arrêts du Conseil d'État, toujours favorables, d'une part à la juridiction du prêtre dans son église, d'autre part à la hiérarchie catholique canonique contre les schismatiques. Enfin, à plus long terme, le dialogue renoué entre le Saint-Siège et le gouvernement français à partir de 1921 aboutit en 1924 à la solution des associations diocésaines. En réalité les rapports conflictuels entre l'Église et l'État ont cessé dès 1914, avec la mort de Pie X et avec la guerre. La période 1905-1914 est essentielle pour comprendre la construction du compromis durable entre l'Église et l'État. La réalité des rapports entre l'Église et l'État ayant été réglée par un ajustement progressif du gouvernement face au refus catholique de la loi de 1905, celle-ci, pour tout ce qui a trait aux catholiques - car il en va différemment pour les protestants et les juifs - peut être considérée comme un texte purement théorique qui n'a jamais trouvé de véritable 
application. Quant aux aménagements postérieurs, n'ont-ils pas fini par constituer une sorte de nouveau concordat?

\section{NOTES}

1.. Émile COMBES considère que « la seule voie restée libre aux deux pouvoirs en conflit, c'est la voie ouverte aux époux mal assortis : le divorce, et de préférence le divorce par consentement mutuel »; discours d'Auxerre, le 4 septembre 1904.

2.. Georges CLEMENCEAU, L'Aurore, 21 juillet 1904.

3.. Julien DE NARFON, Vers l'Église libre, Paris, Librairie Alcan, 1905, p. 360.

4.. Bruno NEVEU, « Pour une histoire du gallicanisme administratif de l'an IX à nos

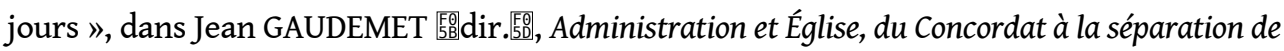
l'Église et de l'État, Genève, Librairie Droz, 1987, p. 58.

5.. Georges CLEMENCEAU, L'Aurore, 21 juillet 1904.

6.. C'est la vision d'Émile Combes, dont le projet de séparation, présenté le 10 novembre 1904 à la Chambre, est rejeté car trop anticlérical.

7.. La vision libérale est défendue par Aristide Briand, (premier rapporteur d'une commission élue en 1903 pour étudier la question de la séparation) puis par M. BienvenuMartin, ministre de l'Instruction, des Beaux-Arts et des cultes au moment du vote de la loi.

8.. Gérard CHOLVY et Yves-Marie HILAIRE, Histoire religieuse de la France contemporaine, 1880-1930, Toulouse, Éditions Privat, 1986, tome 2, p. 107.

9.. Julien DE NARFON, La Séparation des Églises et de l'État, origines, étapes, bilan, Paris, Librairie Alcan, 1912, p. 212.

10.. Jean GAUDEMET, « Une instance de liaison. Le conseiller pour les affaires religieuses auprès du ministre des relations extérieures ", dans Jean GAUDEMET [5: Administration et Église..., ouv. cité, p. 48.

11.. « Impossible... », dans La Semaine religieuse du diocèse de Meaux (Semaine relig. Meaux), 18-25 juin 1910, p. 299.

12.. «Le petit concordat », dans La Semaine religieuse de la ville et du diocèse de Versailles ( Semaine relig. Versailles), $1^{\mathrm{er}}-7$ octobre 1905, p. 138.

13.. «La séparation et les associations paroissiales », dans Semaine relig. Meaux, 6-13 août 1905, p. 374.

14.. « Le petit concordat », dans Semaine relig. Versailles, art. cité.

15.. « La Séparation de l'Église et de l'État », dans Semaine relig. Versailles, 9-16 juillet 1905, p. 780 ; « La Séparation et les associations paroissiales », dans Semaine relig. Meaux, 6-13 août 1905, p. 373.

16.. «Concordat et séparation », dans Semaine relig. Versailles, 26-31 mars 1905, p. 542.

17.. « Société populaire d'Économie sociale », dans Semaine relig. Meaux, 30 avril - 7 mai

1905, p. 210 ; «Ce qu'est la Séparation aux États-Unis », dans Semaine relig. Versailles, 31 décembre 1905-6 janvier 1906, p. 347.

18.. « La Séparation de l'Église et de l'État », dans Semaine relig. Versailles, 9-16 juillet 1905, p. 780 . 
19.. "La pétition contre la séparation dans le diocèse ", dans Semaine relig. Meaux, 16-23 juillet 1905, p. 329.

20.. « Protestation des membres du conseil de fabrique de la paroisse Notre-Dame de Versailles ", dans Semaine relig. Versailles, 9-15 juillet 1905, p. 778.

21.. « La loi de séparation », dans Semaine relig. Versailles, 26 novembre-2 décembre 1905, p. 265.

22.. «Le petit concordat », dans Semaine relig. Versailles, ${ }^{\mathrm{er}}-7$ octobre 1905, p. 138.

23.. "Lettre circulaire sur les associations paroissiales", dans Semaine relig. Versailles, 3-10 décembre 1905, p. 277.

24.. « Les projets de M. Briand », dans Semaine relig. Meaux, 28 août-5 septembre 1909, p. 518.

25.. Ibidem.

26.. « Une manœuvre de M. Briand », dans Semaine relig. Meaux, 4-11 septembre 1909, p. 528 .

27.. "Lettre pastorale de Mgr l'évêque de Meaux ", dans Semaine relig. Meaux, 28 mai-3 juin 1910, p. 259.

28.. « Impossible... », dans Semaine relig. Meaux, 18-25 juin 1910, p. 299.

29.. « Le Criterium », dans Semaine relig. Meaux, 18-25 juin 1910, p. 301.

30.. « C'est la loi », dans Semaine relig. Versailles, 29 juillet-5 août 1906, pp. 191-192 ; « Lois intangibles », Semaine relig. Meaux, 10-17 mars 1907, p. 117.

31. Bruno NEVEU, « Pour une histoire du gallicanisme administratif », art. cité, p. 86.

32. Dominique LE TOURNEAU, L'Église et l'État en France, Paris, Presses Universitaires de France, 2000, p. 103.

33.. Jean KERLEVEO, L'Église catholique en régime français de séparation. Tome 1 : L'occupation des églises par le desservant et les fidèles, Aire sur la Lys, Éditeur Jean Mordacq, 1951, p. XV.

34.. Pour l'État lui-même, les deux propositions de la loi créent un paradoxe : une association cultuelle catholique qui serait conforme à la loi de 1905 mais n'obéirait pas à la hiérarchie, serait à la fois schismatique au regard de la discipline catholique, et illégale au regard de la loi de 1905 si l'on considère l'article quatre. Ce type d'association cultuelle schismatique a d'ailleurs été considéré par la jurisprudence du Conseil d'État comme inexistante, puisque ne se conformant pas « aux règles d'organisation générales » de l'Église, donc incapable de recevoir les biens de la fabrique et la jouissance de l'église. Voir Jean KERLEVEO, L'Église catholique en régime français de séparation, ouv. cité.

35.. "Les associations de laïcs selon le Droit ecclésiastique », dans Semaine relig. Versailles, 19-26 mars 1905, p. 521. Voir aussi Raoul ALLIER, « La Séparation des Églises et de l'État, l'Enquête du Siècle ", dans Cahiers de la Quinzaine, $14{ }^{\text {ème }}$ cahier de la $6{ }^{\text {ème }}$ série, Paris, 1905. 36.. Jacques GADILLE et Jean-Marie MAYEUR, Libéralisme, industrialisation, expansion européenne. 1830-1914, dans Jean-Marie MAYEUR, Charles et Luce PIETRI, André VAUCHEZ et Marc VENARD [dir.], Histoire du Christianisme, Paris, Éditions Desclée, 1990-, tome 11, 1995, p. 529.

37.. « De tout un peu », dans Semaine relig. Meaux, 17-24 décembre 1905, p. 611.

38. Seuls deux évêques, contre 72 , approuvèrent le principe de la loi !

39.. " Lettre des cardinaux français à Monsieur Loubet ", dans Semaine relig. Versailles, 2-9 avril 1905, p. 549 ; « La séparation et les associations paroissiales », dans Semaine relig. Meaux, 6-13 août 1905, p. 375.

40.. « Lettre des cardinaux français... », art. cité, p. 549.

41.. " La séparation et les associations paroissiales ", dans Semaine relig. Meaux, 6-13 août 1905, p. 375. 
42.. Jean-Marie MAYEUR, « Des catholiques libéraux devant la loi de séparation ; les cardinaux verts ", dans Religion et politique ; les deux guerres mondiales; histoire de Lyon et du Sud-Est. Mélanges offerts à André Latreille, Collection du Centre d'histoire du catholicisme de l’Université Lyon 2 n$^{\circ}$ 10, Lyon, Éditions Audin, 1972, pp. 207-224.

43.. Remarquons que, même en acceptant les associations cultuelles, l'Église aurait pratiquement tout perdu : la jouissance de tous les immeubles pris à l'Église de France pendant la Révolution et dont l'usage lui avait été rendu après la Concordat, la jouissance gratuite des évêchés au bout de deux ans, celle des séminaires et presbytères au bout de cinq ans, le traitement du clergé, les fondations pieuses antérieures au Concordat.

44.. Aristide Briand veut mettre l'Église catholique « dans l'impossibilité de sortir de la loi ", déclaration au Sénat, séance du 28 décembre 1906.

45.. Michel BAZOCHE, Le régime légal des cultes en France, Paris, Publications administratives, 1948, p. 12.

46.. « La déclaration des évêques de France », dans Semaine relig. Meaux, 3-10 février 1907, p. 50.

47.. « Melun », dans Semaine relig. Meaux, 24 février-3 mars 1907, p. 89.

48.. «La situation », dans Semaine relig. Meaux, 10-17 mars 1907, p. 113.

49.. Semaine relig. Meaux, 17-24 mars 1907, p. 121.

50.. « Réflexions sur la location des églises », dans Semaine relig. Versailles, 11-18 juin 1905, p. 714.

51.. « Hier et aujourd'hui », dans Semaine relig. Meaux, 24-31 mars 1907, p. 143.

52.. Lettre du sous-préfet de Fontainebleau au préfet de Seine-et-Marne, Archives départementales de Seine-et-Marne, $2 Q 231$.

53. « La hiérarchie catholique et la cour de cassation », dans Semaine relig. Meaux, 4-11 mai 1912, p. 280-281.

54.. Ministre de l'Intérieur dans le gouvernement Sarrien puis président du Conseil à partir du 5 novembre 1906.

55.. Expiration du délai fixé pour la formation des associations cultuelles.

56.. « À propos de l'exercice du culte dans nos églises », dans Semaine relig. Versailles, 23-30 décembre 1906, p. 454.

57.. Rappelons que les députés du département ont voté la loi de séparation à l'unanimité.

58.. C'est le cas à Nanteuil-les-Meaux, à l'insu du curé ; « Démenti au Petit Parisien », dans Semaine relig. Meaux, 23-30 décembre 1906, p. 623.

59.. " Communication de l'évêché », dans Semaine relig. Meaux, 23-30 décembre 1906, p. 622.

60.. Semaine relig. Meaux, 16-23 décembre 1906, p. 610.

61.. « Devant la justice de paix », dans Semaine relig. Meaux, 10-17 février 1907, p. 70.

62.. «Une consultation », dans Semaine relig. Meaux, 3-10 mars 1907, p. 103.

63.. Roger AUBERT [dir.], L'Église dans le monde moderne : de 1848 à nos jours, dans Roger AUBERT, David KNOWLES et Ludovicus Jacobus ROGIER [dir.], Nouvelle Histoire de l'Église, Paris, Éditions du Seuil, 1963-, tome 5, 1975, p. 87.

64.. " La séparation et les associations paroissiales ", dans Semaine relig. Meaux, 6-13 août 1905, p. 375.

65.. Ibidem.

66.. «Lettre circulaire », dans Semaine relig. Versailles, 12-19 mars 1905, p. 502.

67.. Bruno NEVEU, « Pour une histoire du gallicanisme administratif... », art. cité, p. 96. 


\section{RÉSUMÉS}

La loi de séparation de 1905 pose le principe de l'indépendance absolue entre État et Églises. On constate néanmoins qu'il est impossible pour l'Église catholique et l'État de s'ignorer mutuellement. Au cours de l'élaboration du projet de loi, s'opposent les libéraux, partisans d'une autonomie totale de l'Église, et les héritiers d'un gallicanisme concordataire, soucieux de maintenir un certain contrôle sur l'Église. La loi de 1905, si elle a pu être ressentie par les catholiques comme une nouvelle persécution, au sortir d'une période marquée par l'anticléricalisme républicain, constitue en réalité le début d'un processus de pacification, que l'on peut considérer comme un nouveau concordat, entre l'Église et l'État. Face à l'unité des catholiques qui, obéissant au pape, choisissent de rester hors-la-loi, le gouvernement français doit composer. Il élabore de nouvelles lois permettant aux catholiques d'exercer leur culte dans la liberté et la légalité. Des positions radicales de chacune des deux parties, seule l'Église, forte de son unité, a pu tenir la sienne. L'Église est ainsi passée du statut de victime de la loi de séparation, à celui de partenaire dans l'élaboration d'un nouveau compromis. En ce qui concerne l'Église catholique, la loi de 1905 peut être considérée comme un texte purement théorique qui n'a jamais trouvé de véritable application.

The legal separation of the Church and the State in 1905: an impossible break-up

The 1905 law of separation set the principle of a radical independence between the State and the Church. But it was impossible for Church and State to ignore each other. The elaboration of the law led to an opposition between the liberals who wanted total independence for the Catholic Church and the successors of gallicanisme who wanted to keep Church under state control. The 1905 law, experienced by the Catholics as a new persecution after a time of republican anticlericalism, really represented the first step of a pacification process, a new kind of concordat. The Church, siding with the pope, rejected the law. The government therefore had to frame new laws allowing Catholics to have a legal and free practice of their religion. In the conflict between the State and the Church, only the Church, thanks to its unity, was able to stick to its position. Initially the victim of the law, the Church managed to become an actor in the framing of a compromise. Regarding the Catholic Church, the law of 1905 just seemed a theoretical document which never found its genuine implementation.

\section{AUTEUR}

MATHILDE GUILBAUD

agrégée d'histoire et doctorante à l'Université Paris 13 